\title{
Relativistic Coulomb scattering of spinless bosons
}

\author{
M. G. Garcia* and A. S. de Castrdt
}

(Dated: 11 February 2015)

\begin{abstract}
The relativistic scattering of spin-0 bosons by spherically symmetric Coulomb fields is analyzed in detail with an arbitrary mixing of vector and scalar couplings. It is shown that the partial wave series reduces the scattering amplitude to the closed Rutherford formula exactly when the vector and scalar potentials have the same magnitude, and as an approximation for weak fields. The behavior of the scattering amplitude near the conditions that furnish its closed form is also discussed. Strong suppressions of the scattering amplitude when the vector and scalar potentials have the same magnitude are observed either for particles or antiparticles with low incident momentum. We point out that such strong suppressions might be relevant in the analysis of the scattering of fermions near the conditions for the spin and pseudospin symmetries. From the complex poles of the partial scattering amplitude the exact closed form of bound-state solutions for both particles and antiparticles with different scenarios for the coupling constants are obtained. Perturbative breaking of the accidental degeneracy appearing in a pair of special cases is related to the nonconservation of the Runge-Lenz vector.
\end{abstract}

PACS numbers: 25.80.Dj, 03.65.Pm, 11.80.-m, 03.65.Ge

* marcelogarcia82@gmail.com

$\dagger$ castro@pq.cnpq.br 


\section{INTRODUCTION}

Elastic scattering experiments of a nucleon or electron by atomic nuclei is of the greatest importance because they can furnish details of the current and charge distributions within the nucleus by measuring the deviation of the actual scattering from that due to a point nucleus. The nonrelativistic quantum solution for the elastic scattering of particles in Coulomb fields furnishes the classical Rutherford scattering amplitude. This result was first obtained by Gordon [1] via separation in parabolic coordinates. Then, Mott found the partial wave series in terms of phase shifts by using the Schrödinger equation [2] as well as the Dirac equation with the Coulomb field as a time component of a Lorentz vector [3]. In this last case, though, the scattering amplitude is not known in a closed form.

The elastic scattering of a spinless particle such as a pion or a kaon by a point nucleus is also of fundamental importance and this problem has received some attention in the literature. Kang and Brown [4] approached the scattering amplitude by using the KleinGordon (KG) equation with the time component of a Coulomb field and found a perturbative expansion on the coupling constant to the third order whereas Hetherington [5] analyzed questions regarding the convergence of the partial wave series, and Rawitscher [6] examined deviations of the $\mathrm{KG}$ from the Schrödinger scattering for small velocities. Omission of a term involving the square of the field in the KG equation has been repeated often [7], and in another publication the contribution of that term only appeared in the lowest-order terms of the partial wave series [8]. Latter, Cooper, Jeppesen and Johnson [9] filled this gap with an analytic approximate expression taking into account arbitrarily high angular momentum. Experimental data from the scattering of kaons by nuclei were analyzed by Hill, Hetherington and Ravenhall [10] using the KG equation with a vector Coulomb field plus a scalar Woods-Saxon field. Jansen, Pusch and Soff [11] investigated the continuum solutions of the KG equation with vector and scalar couplings for a number of fields, including the Coulomb field, but their work was not concerned with the scattering amplitude problem. As a matter of fact, the bound-state solutions had already received attention before [12] (see also Ref. [13]). The mixed vector-scalar Coulomb field was also approached in arbitrary dimensions with full attention to the bound states [14], and later to the scattering states and their phase shifts [15]. Even so, the authors of Ref. [15] did not examine in detail the full properties of the scattering amplitude and for that reason they missed the opportunity 
to explore some of its important attributes.

Physical systems with fermions subject to vector and scalar fields are not uncommon in the literature. The Dirac equation with vector and scalar fields has been originally used in an attempt to describe the dynamics between a quark and antiquark in order to calculate meson masses [16]. Later, the mixing vector-scalar was used for investigating the implications of a tiny contribution of the scalar Coulomb potential to atomic spectroscopy [17]. The nucleonnucleus scattering has been described quite well with strong repulsive vector and attractive scalar fields since 1980s (see e.g. [18] and references therein). The Dirac Hamiltonian with vector and scalar fields with couplings of equal magnitude is invariant under a $\mathrm{SU}(2)$ algebra [19] and vector and scalar fields with near equality in the magnitudes have been used as nuclear mean-field potentials [20] (related to the pseudospin symmetry), and in the quark model [21] as well as for considering the possibility of antifermions in nuclei [22] (related to the spin symmetry). Indeed, most studies of spin and pseudospin symmetries are concerned with bound states, and it seems that the experimental data on nucleon-nucleus scattering does not exhibit the features of the approximate pseudospin symmetry [20], [23],[24]. The authors of Ref. [24] suggest that the long-range nature of the Coulomb potential is responsible for breaking the pseudospin symmetry. Nevertheless, the approximate realization of that symmetry has been found by other authors using different kinds of analysis [25]. Recently, the perturbative nature of both symmetries with Coulomb fields were discussed in Ref. [26] regarding bound states. It has also been shown that, for fields of any shape, the conditions on the coupling constants that originate the pseudospin and spin symmetries make spin-1/2 and spin-0 particles to have the same energy spectrum due to the disappearance of the spin-orbit coupling and the Darwin terms of either the upper component (spin symmetry) or the lower component (pseudospin symmetry) of the Dirac spinor [27]. It is the absence of the spin-orbit term for one of the components of the Dirac spinor that explains the existence of quasi-degenerate pseudospin doublets in certain nuclei, and the existence of nearly degenerate spin doublets in heavy-light quark meson.

In applications, the scattering of mesons by a nucleus is described by a short-range field and an additional vector Coulomb field $V_{v}=\hbar c Z \alpha / r$ acting outside the region of the nuclear matter ( $Z$ the product of the nuclear and mesonic charges, and $\alpha$ the fine-structure constant). Addition of a short-range scalar field, related to the exchange of mediating massive bosons, is important not only for scattering states but also for describing the spectrum of a meson 
immersed in a nuclear environment.

The purpose of this work is to consider the partial wave analysis for the relativistic elastic scattering of spin-0 bosons by a Coulomb field in the presence of mixing of vector and scalar couplings. The unified analysis of the KG equation with vector and scalar Coulomb fields allows one to find the conditions under which closed forms for the scattering amplitude can be found that apply to both scattering states and bound states. The addition of a scalar coupling to the usual vector coupling increases its interest because the partial wave series can be exactly summed when the vector and scalar couplings have the same magnitude. The Rutherford formula can also be retrieved as an approximation for very small coupling constants, as it happens in the nonrelativistic limit of the theory. The solution of the KG equation is expressed in terms of the Whittaker functions, and the scattering amplitude for small pure vector and pure scalar couplings, as well as the small deviation from the exact formula for vector and scalar couplings with the same magnitude, is calculated in a perturbative way. The exact bound-state solutions for a restrict range of coupling constants are obtained from the complex poles of the partial scattering amplitude. For such bound states the eigenenergies are expressed in terms of solutions of a second-degree algebraic equation and the eigenfunctions are expressed in terms of the generalized Laguerre polynomials. For both kinds of stationary states we present a detailed study of some interesting particular cases. Surprisingly, there are strong suppressions of the scattering amplitude when the vector and scalar fields have equal magnitude either for particles or antiparticles with low incident momentum. Furthermore, we show that the accidental degeneracy for bound states appearing in the nonrelativistic limit and when the vector and scalar couplings have the same magnitude, related to the conservation of the Runge-Lenz vector, is broken perturbatively. Finally, we suggest that the strong suppressions mentioned above might be relevant in the analysis of the scattering of fermions and antifermions near the conditions required for the spin and pseudospin symmetries. 


\section{VECTOR AND SCALAR COUPLINGS IN THE KLEIN-GORDON EQUA- TION}

The time-independent KG equation for a spinless particle with mass $m$ and energy $E$ under the influence of external vector $\left(V_{v}\right)$ and scalar $\left(V_{s}\right)$ fields reads

$$
\left[(\hbar c)^{2} \nabla^{2}+\left(E-V_{v}\right)^{2}-\left(m c^{2}+V_{s}\right)^{2}\right] \phi=0
$$

Notice that the scalar field is coupled to the mass in accordance with the substitution $m \rightarrow m+V_{s} / c^{2}$. This prescription allows the analysis of repulsive as well attractive scalar couplings in the same framework and furnishes the proper nonrelativistic limit of the KG equation (fields small compared to $m c^{2}$ and $E \simeq m c^{2}$, and an effective field $V_{v}+V_{s}$ ), in contrast with the rule $m^{2} \rightarrow m^{2}+V_{s}^{2} / c^{4}$ employed in [11]-[13]. If one considers spherically symmetric fields then $\phi(\vec{r})$ can be factorized as

$$
\phi_{\nu l m_{l}}(\vec{r})=\frac{u_{\nu}(r)}{r} Y_{l m_{l}}(\theta, \varphi)
$$

where $Y_{l m_{l}}(\theta, \varphi)$ is the usual spherical harmonic, $\nu$ denotes the principal quantum number plus other possible quantum numbers, and $u_{\nu}(r)$ obeys the radial equation

$$
\begin{array}{r}
\frac{d^{2} u_{\nu}}{d r^{2}}+\left[\frac{V_{v}^{2}-V_{s}^{2}-2\left(E V_{v}+m c^{2} V_{s}\right)}{(\hbar c)^{2}}\right. \\
\left.-\frac{l(l+1)}{r^{2}}+k^{2}\right] u_{\nu}=0
\end{array}
$$

in which $\hbar c k=\sqrt{E^{2}-m^{2} c^{4}}$. It may well be worthwhile to note that the spectrum changes its sign if $V_{v}$ does, and that it is symmetrical about $E=0$ if $V_{v}=0$. Notice also that the spectrum presents a degeneracy of order $2 l+1$ with respect to $m_{l}$ due to the spherical symmetry of the fields. Because $\nabla^{2}(1 / r)=-4 \pi \delta(\vec{r})$, unless the fields contain a delta function at the origin, one must impose the homogeneous Dirichlet condition $u_{\nu}(0)=0$ [28]. On the other hand, if both fields vanish at large distances the solution $u_{\nu}$ has the asymptotic behavior $e^{i k r}$ as $r \rightarrow \infty$. Therefore, scattering states only occur if $|E|>m c^{2}$ $(k \in \mathbb{R})$ whereas bound states might occur only if $|E|<m c^{2}(k=i|k|)$.

\section{COULOMB FIELDS}

Antecipating possible future physics applications, a set of mathematical conditions will be explored below. When the vector and scalar fields are of Coulomb type, i.e., they are of 
the form $V_{v}=\hbar c g_{v} / r$ and $V_{s}=\hbar c g_{s} / r$, the use of the abbreviations

$$
\gamma_{l}=\sqrt{(l+1 / 2)^{2}+g_{s}^{2}-g_{v}^{2}}, \quad \eta=\frac{E g_{v}+m c^{2} g_{s}}{\hbar c k}
$$

and the change $\zeta=-2 i k r$ allow to write the radial $\mathrm{KG}$ equation in form of the Whittaker equation

$$
\frac{d^{2} u_{\nu}}{d \zeta^{2}}+\left(-\frac{1}{4}-\frac{i \eta}{\zeta}+\frac{1 / 4-\gamma_{l}^{2}}{\zeta^{2}}\right) u_{\nu}=0
$$

with linearly independent solutions $M_{-i n . \gamma_{l}}(\zeta)$ and $W_{-i \eta, \gamma_{l}}(\zeta)$ behaving like $\zeta^{1 / 2+\gamma_{l}}$ and $\zeta^{1 / 2-\gamma_{l}}$ close to the origin, respectively [29]. Because $u_{\nu}(0)=0$, one has to consider the solution $u_{\nu}$ proportional to [29]

$$
M_{-i \eta, \gamma_{l}}(\zeta)=e^{-\zeta / 2} \zeta^{1 / 2+\gamma_{l}} M\left(1 / 2+\gamma_{l}+i \eta, 1+2 \gamma_{l}, \zeta\right)
$$

Here,

$$
\left|g_{v}\right|<\sqrt{1 / 4+g_{s}^{2}}
$$

and $M(a, b, z)$ is the confluent hypergeometric function

$$
M(a, b, z)=\sum_{j=0}^{\infty} \frac{\Gamma(a+j) \Gamma(b)}{\Gamma(b+j) \Gamma(a)} \frac{z^{j}}{j !}
$$

Using the asymptotic behavior of $M(a, b, z)$ for large $|z|$ and $-\frac{3 \pi}{2}<\arg z \leq-\frac{\pi}{2}[29]$

$$
M(a, b, z) \simeq \frac{\Gamma(b)}{\Gamma(b-a)} e^{-i \pi a} z^{-a}+\frac{\Gamma(b)}{\Gamma(a)} e^{z} z^{a-b}
$$

one can show that for very large $r(|k| r>>1)$

$$
u_{\nu}(r) \simeq \sin \left(k r-l \pi / 2+\delta_{l}\right)
$$

with the relativistic Coulomb phase shift $\delta_{l}=\delta_{l}(\eta)$ given by

$$
\delta_{l}=\frac{\pi}{2}\left(l+1 / 2-\gamma_{l}\right)+\arg \Gamma\left(1 / 2+\gamma_{l}+i \eta\right)
$$

It is instructive to note that the term $V_{v}^{2}-V_{s}^{2}$ in the $\mathrm{KG}$ equation (11) gives rise to an attractive (repulsive) short-range $r^{-2}$ term if one uses the Coulomb field with $\left|V_{v}\right|<\left|V_{s}\right|$ $\left(\left|V_{v}\right|>\left|V_{s}\right|\right)$. Notice also that this short-range term does not exist if the vector and scalar fields have equal magnitude and loses its importance in the limit of small coupling constants. Only in the absence of the short-range term the Runge-Lenz vector is a constant of motion [30]. 


\section{A. Scattering states}

For scattering states, the solution of the KG equation (11) has the asymptotic form

$$
\phi(\vec{r}) \simeq e^{i k r \cos \theta}+f(\theta, \varphi) \frac{e^{i k r}}{r}
$$

where the first term represents a plane wave moving along the direction $\theta=0$ toward the scatterer, and the second represents a radially outgoing wave. For spherically symmetric scatterers, both terms exhibit cylindrical symmetry about the direction of incidence in such a way that $\phi$ and $f$ are independent of $\varphi$. The connection between the forms (2) and (12) allows us to write the scattering amplitude as a partial wave series

$$
f(\theta)=\sum_{l=0}^{\infty}(2 l+1) f_{l} P_{l}(\cos \theta)
$$

where $P_{l}$ is the Legendre polynomial of order $l$ and the partial scattering amplitude is $f_{l}=\left(e^{2 i \delta_{l}}-1\right) /(2 i k)$. With the phase shift (11), up to a logarithmic phase inherent to the Coulomb field, one finds

$$
2 i k f_{l}=-1+e^{i \pi\left(l+1 / 2-\gamma_{l}\right)} \frac{\Gamma\left(1 / 2+\gamma_{l}+i \eta\right)}{\Gamma\left(1 / 2+\gamma_{l}-i \eta\right)}
$$

The series (13) can be summed when $\gamma_{l}=l+1 / 2$ the closed form being [2] (see also [31])

$$
f(\theta)=-\eta \frac{\Gamma(1+i \eta)}{\Gamma(1-i \eta)} \frac{\exp \left(-i \eta \ln \sin ^{2} \theta / 2\right)}{2 k \sin ^{2} \theta / 2}, \quad \theta \neq 0
$$

which gives the well-known Rutherford scattering formula for the differential cross section in classical and nonrelativistic quantum mechanics. It is worthwhile to note that this last equation is exact if one considers $\left|g_{v}\right|=\left|g_{s}\right|$. Also, it is appropriate as an approximation for small coupling constants.

In order to study the behavior of the scattering amplitude near the conditions that furnish its closed form, we will perform the sum (13) for small $g_{v}$ and $g_{s}$, convenient for exploring the nonrelativistic limit. The expansion near the condition $\left|g_{v}\right|=\left|g_{s}\right|$ is made easier if one defines $g_{\Delta}$ and $g_{\Sigma}$ by

$$
g_{\Delta}=g_{v}-g_{s}, \quad g_{\Sigma}=g_{v}+g_{s}
$$

The expansions of the sum (13) out to next-to-leading order can be carried out by using a pair of properties of the gamma function [29]: $\Gamma(1+z)=z \Gamma(z)$ and $1 / \Gamma(z) \simeq z e^{\gamma_{e} z}$ for $z \simeq 0$, where $\gamma_{e}$ is Euler's constant. In addition, we use a few mathematical identities taken from Ref. [4], viz. 


$$
\begin{aligned}
\sum_{l=0}^{\infty} P_{l}(\cos \theta) & =\frac{1}{2 \sin \theta / 2} \\
\sum_{l=0}^{\infty}(2 l+1) P_{l}(\cos \theta) & =0, \quad \theta \neq 0 \\
\sum_{l=0}^{\infty}(2 l+1) P_{l}(\cos \theta) \psi(l+1) & =-\frac{1}{2 \sin ^{2} \theta / 2} \\
\sum_{l=0}^{\infty}(2 l+1) P_{l}(\cos \theta) \psi^{2}(l+1) & =\frac{\ln \sin \theta / 2+\gamma_{e}}{\sin ^{2} \theta / 2} \\
\sum_{l=0}^{\infty}(2 l+1) P_{l}(\cos \theta)\left[\psi^{(2)}(l+1)+4 \psi^{3}(l+1)\right] & =-6\left(\frac{\ln \sin \theta / 2+\gamma_{e}}{\sin \theta / 2}\right)^{2}
\end{aligned}
$$

where $\psi(z)=d \ln \Gamma(z) / d z$ is the digamma (psi) function and $\psi^{(2)}(z)=d^{2} \psi(z) / d z^{2}$. With such identities, comparison of the expansion of (15) and (13) with $f_{l}$ given by (14) furnishes the desired expansions.

The expansions for $\left|g_{v}\right|<<1$ and $\left|g_{s}\right|<<1$ are given by

$$
f(\theta)= \begin{cases}-\frac{g_{v} E}{2 \hbar c k^{2} \sin ^{2} \theta / 2} e^{-i \frac{2 g_{v} E\left(\ln \sin \theta / 2+\gamma_{e}\right)}{\hbar c k}} f_{v}, & g_{s}=0 \\ -\frac{g_{s} m c^{2}}{2 \hbar c k^{2} \sin ^{2} \theta / 2} e^{-i \frac{2 g_{s} m c^{2}\left(\ln \sin \theta / 2+\gamma_{e}\right)}{\hbar c k}} f_{s}, & g_{v}=0\end{cases}
$$

with

$$
\begin{aligned}
& f_{v}=1-\frac{g_{v} \pi \hbar c k \sin \theta / 2}{2 E}+\mathcal{O}\left(g_{v}^{2}\right) \\
& f_{s}=1+\frac{g_{s} \pi \hbar c k \sin \theta / 2}{2 m c^{2}}+\mathcal{O}\left(g_{s}^{2}\right)
\end{aligned}
$$

The leading terms in both expansions are consistent with the nonrelativistic limit with $|E| \simeq m c^{2}$ and the next-to-leading order contributes for changing the angular distribution. Differences between vector and scalar couplings appear even in the zeroth-order terms. A remarkable difference between the natures of vector and scalar couplings appears in the next-to-leading order: the pure scalar coupling always contributes to increase (decrease) $|f(\theta)|$ when the the scalar field is repulsive (attractive), as for the the pure vector coupling, though, $|f(\theta)|$ increases or decreases depending on the sign of $g_{v} E$. 
Near the condition $\left|g_{v}\right|=\left|g_{s}\right|$, with $g_{\Delta}$ and $g_{\Sigma}$ defined in (16), one obtains the following perturbative approximations

$$
f(\theta)= \begin{cases}-\frac{g_{\Sigma}\left(E+m c^{2}\right)}{4 \hbar c k^{2} \sin ^{2} \theta / 2} e^{-i \frac{g_{\Sigma}\left(E+m c^{2}\right)\left(\ln \sin \theta / 2+\gamma_{e}\right)}{\hbar c k}} f_{\Sigma}, & g_{\Delta}=0 \\ -\frac{g_{\Delta}\left(E-m c^{2}\right)}{4 \hbar c k^{2} \sin ^{2} \theta / 2} e^{-i \frac{g_{\Delta}\left(E-m c^{2}\right)\left(\ln \sin \theta / 2+\gamma_{e}\right)}{\hbar c k}} f_{\Delta}, & g_{\Sigma}=0\end{cases}
$$

with

$$
\begin{aligned}
& f_{\Sigma}=1-\frac{1}{2}\left[\frac{g_{\Sigma}\left(E+m c^{2}\right)\left(\ln \sin \theta / 2+\gamma_{e}\right)}{\hbar c k}\right]^{2}+\mathcal{O}\left(g_{\Sigma}^{3}\right) \\
& f_{\Delta}=1-\frac{1}{2}\left[\frac{g_{\Delta}\left(E-m c^{2}\right)\left(\ln \sin \theta / 2+\gamma_{e}\right)}{\hbar c k}\right]^{2}+\mathcal{O}\left(g_{\Delta}^{3}\right)
\end{aligned}
$$

The leading terms of these last expansions, for $g_{\Delta}=0\left(g_{\Sigma}=0\right)$, reveal consistency with the nonrelativistic limit with $E \simeq+m c^{2}\left(E \simeq-m c^{2}\right)$ and that the scattering amplitude is strongly suppressed when $E \simeq-m c^{2}\left(E \simeq+m c^{2}\right)$ independently of the magnitudes of $g_{v}$ and $g_{s}$. The next-to-leading order terms distort the angular distribution and always contribute to decrease $|f(\theta)|$.

\section{B. Bound states}

If $k=i|k|\left(|E|<m c^{2}\right)$, the partial scattering amplitude becomes infinite when $1 / 2+$ $\gamma_{l}-\operatorname{Im} \eta=-N$, where $N=0,1,2, \ldots$, due to the poles of the gamma function in the numerator of (14). Because $\operatorname{Im} \eta>0$, beyond the constraint (7) one has $g_{s}<\left|g_{v}\right|$. Remembering the asymptotic behavior of the confluent hypergeometric function given by (9), we see that the Whittaker function $M_{\operatorname{Im} \eta, \gamma_{l}}(2|k| r)$ goes asymptotically as $e^{-|k| r}$ as $r$ increases because $M\left(-N, 1+2 \gamma_{l}, 2|k| r\right)$ is proportional to $L_{N}^{\left(2 \gamma_{l}\right)}(2|k| r)$, the generalized Laguerre polynomial of order $N$. Thus, the characteristic pair $\left(E_{\nu}, u_{\nu}\right)$ represents a spatially localized state explicitly expressed as

$$
\begin{gathered}
E_{\nu}=m c^{2} \frac{-\frac{g_{v}}{\nu} \frac{g_{s}}{\nu} \pm\left[1+\left(\frac{g_{v}}{\nu}\right)^{2}-\left(\frac{g_{s}}{\nu}\right)^{2}\right]^{1 / 2}}{1+\left(\frac{g_{v}}{\nu}\right)^{2}} \\
u_{\nu}=A_{\nu} r^{1 / 2+\gamma_{l}} e^{-|k| r} L_{\nu-1 / 2-\gamma_{l}}^{\left(2 \gamma_{l}\right)}(2|k| r)
\end{gathered}
$$

where the quantum number $\nu$ satisfies

$$
\nu=\operatorname{Im} \eta=N+1 / 2+\gamma_{l}>1 / 2
$$


and $A_{\nu}$ is a normalization constant. The energy levels enter from the continuum to the bound-state gap $\left(|E|<m c^{2}\right)$ coming from the upper continuum (related to particle states) or from the lower continuum (related to antiparticle states).

One may check that the nonrelativistic limit is correct. Indeed, if one sets $\left|g_{v}\right|<<1$ and $\left|g_{s}\right|<<1$, one gets

$$
\frac{E_{n}}{m c^{2}} \simeq \pm\left[1-\frac{\left(g_{s} \pm g_{v}\right)^{2}}{2 n^{2}}\right], \quad g_{s} \pm g_{v}<0
$$

in which $n$, contained in the denominator of (25), is a positive integer given by $n=N+$ $l+1=1,2,3, \ldots$ with $l \leq n-1$. Beyond the degeneracy due to the rotational symmetry, a distinguished degeneracy with respect to $l$ results in a spectrum $n^{2}$-fold degenerate.

For $g_{v}=\varepsilon g_{s}\left(g_{s}<0\right)$, the expansion of (22) until next-to-leading order in $\varepsilon$ furnishes

$$
\begin{gathered}
\frac{E_{\nu}}{m c^{2}}= \pm\left[1-\left(g_{s} / \nu\right)^{2}\right]^{1 / 2}-\varepsilon\left(g_{s} / \nu\right)^{2}+\mathcal{O}\left(\varepsilon^{2}\right) \\
\nu=N+1 / 2+\sqrt{(l+1 / 2)^{2}+g_{s}^{2}}+\nu_{\varepsilon}
\end{gathered}
$$

with

$$
\nu_{\varepsilon}=-\varepsilon^{2} \frac{g_{s}^{2}}{2 \sqrt{(l+1 / 2)^{2}+g_{s}^{2}}}+\mathcal{O}\left(\varepsilon^{3}\right)
$$

in such a way that the spectrum is symmetrical about $E=0$ in the case of a pure scalar coupling (the zeroth-order term), as expected. The addition of a small vector coupling increases the upper bound on $\left|g_{s}\right|$, breaks the symmetry of the spectrum and makes the states of particles (antiparticles) more tightly bound than the states of antiparticles (particles) if the vector coupling is attractive (repulsive).

On the other hand, for $g_{s}=\varepsilon g_{v}$ one gets

$$
\begin{gathered}
\frac{E_{\nu}}{m c^{2}}=-\frac{\operatorname{sgn}\left(g_{v}\right)}{\left[1+\left(g_{v} / \nu\right)^{2}\right]^{1 / 2}}-\varepsilon \frac{\left(g_{v} / \nu\right)^{2}}{1+\left(g_{v} / \nu\right)^{2}}+\mathcal{O}\left(\varepsilon^{2}\right) \\
\nu=N+1 / 2+\sqrt{(l+1 / 2)^{2}-g_{v}^{2}}+\nu_{\varepsilon}
\end{gathered}
$$

with

$$
\nu_{\varepsilon}=\varepsilon^{2} \frac{g_{v}^{2}}{2 \sqrt{(l+1 / 2)^{2}-g_{v}^{2}}}+\mathcal{O}\left(\varepsilon^{3}\right)
$$

In this last case, the positive (negative) energy levels are excluded from the spectrum if the vector field is repulsive (attractive) when $\varepsilon=0$, and the energies with the least absolute 
values tend to $-m c^{2} \operatorname{sgn}\left(g_{v}\right) / \sqrt{2}$ when $\left|g_{v}\right|$ tends to its limit value equal to $1 / 2$ in such a way that particles (antiparticles) are always associated with positive (negative) energy levels. The addition of a scalar contaminant improves the upper bound imposed on $\left|g_{v}\right|$ and increases (decreases) the binding energy if $\varepsilon<0(\varepsilon>0)$. The difference between bosons and fermions is quite remarkable. Whereas fermions dive into the continuum of negative energies when they are under the influence of a pure strong and attractive vector Coulomb field ( $E=-m c^{2}$ for $\left.g_{v}=Z \alpha=-1\right)$ and pair creation has to be considered, the eigenenergy for bosons takes on a real value only if $g_{v}=Z \alpha<1 / 4$.

In order to study the spectrum near the condition $\left|g_{v}\right|=\left|g_{s}\right|$, necessarily with $g_{s}<0$, we will perform an expansion of (22) and (24) for small $g_{\Delta}$ and $g_{\Sigma}$ defined in (16). The dependence of $\nu$ on $g_{\Delta}$ and $g_{\Sigma}$ comes through $g_{\Delta} g_{\Sigma}$ so that the expansion for $\nu$ is given by

$$
\nu=N+l+1-\frac{g_{\Delta} g_{\Sigma}}{2 l+1}+\mathcal{O}\left(g_{\Delta}^{2} g_{\Sigma}^{2}\right)
$$

For small $g_{\Delta}$ one gets

$$
\begin{aligned}
& \frac{E_{\nu}^{(+)}}{m c^{2}}=1-\frac{2\left(g_{\Sigma} / 2 \nu\right)^{2}}{1+\left(g_{\Sigma} / 2 \nu\right)^{2}}+\varepsilon_{\Delta} \\
& \frac{E_{\nu}^{(-)}}{m c^{2}}=-1+g_{\Delta}^{2} \frac{1}{2 \nu^{2}}+\mathcal{O}\left(g_{\Delta}^{3}\right)
\end{aligned}
$$

with

$$
\varepsilon_{\Delta}=g_{\Delta} \frac{2\left(g_{\Sigma} / 2 \nu\right)^{3}}{\nu\left[1+\left(g_{\Sigma} / 2 \nu\right)^{2}\right]^{2}}+\mathcal{O}\left(g_{\Delta}^{2}\right)
$$

whereas for small $g_{\Sigma}$ one has

$$
\begin{gathered}
\frac{E_{\nu}^{(+)}}{m c^{2}}=1-g_{\Sigma}^{2} \frac{1}{2 \nu^{2}}+\mathcal{O}\left(g_{\Sigma}^{3}\right) \\
\frac{E_{\nu}^{(-)}}{m c^{2}}=-1+\frac{2\left(g_{\Delta} / 2 \nu\right)^{2}}{1+\left(g_{\Delta} / 2 \nu\right)^{2}}+\varepsilon_{\Sigma}
\end{gathered}
$$

with

$$
\varepsilon_{\Sigma}=-g_{\Sigma} \frac{2\left(g_{\Delta} / 2 \nu\right)^{3}}{\nu\left[1+\left(g_{\Delta} / 2 \nu\right)^{2}\right]^{2}}+\mathcal{O}\left(g_{\Sigma}^{2}\right)
$$

Here, the accidental degeneracy already observed in the nonrelativistic limit also comes to the scene in the zeroth-order of the expansions in $g_{\Delta}$ for $E_{\nu}^{(+)}$, and $g_{\Sigma}$ for $E_{\nu}^{(-)}$. In this particular order, there is no upper bound on the coupling constant and for an attractive (repulsive) 
vector field the energy levels emerge from the upper (lower) continuum for small couplings and tend asymptotically to the lower (upper) continuum for large couplings. The accidental degeneracy in the nonrelativistic Coulomb problem is related to the conservation of the Runge-Lenz vector [30]. The term $V_{v}^{2}-V_{s}^{2}$ in the KG equation (11), due to its accompanying $r^{-2}$ term, violates the conservation of the Runge-Lenz vector. $E_{\nu}^{(+)}$in (33) and $E_{\nu}^{(-)}$in (37) reveal that the breaking of the accidental degeneracy is perturbative. This can be seen from the fact that $g_{\Delta}$ and $g_{\Sigma}$ act as perturbative parameters in the above equations, so that one can go continuously from a bound state without accidental degeneracy to a bound state with accidental degeneracy (the nonperturbed state) as these parameters go to zero. This does not happen for $E_{\nu}^{(-)}$in (34) nor for $E_{\nu}^{(+)}$in (36) due to the inexistence of bound states in the zeroth-order. Related to the perturbative breaking of the accidental degeneracy, a worthwhile investigation using the Runge-Lenz vector as a perturbative method for the classical scattering in a perturbed Coulomb field can be found in Ref. [32].

\section{FINAL REMARKS}

The partial wave analysis for the elastic scattering of spin-0 bosons by a Coulomb field with a general mixing of vector and scalar couplings was done in detail. Not only in the nonrelativistic limit of the theory does the partial-wave series for the scattering amplitude reduce to that one giving the Rutherford formula but also when the vector and scalar fields have the same magnitude. We calculated the scattering amplitude for small pure vector and pure scalar couplings, as well as deviations from the exact formula for vector and scalar couplings with the same magnitude, in a perturbative way. The complex poles of the partial scattering amplitude furnished the exact bound-state solutions. The eigenenergies of such bound states are solutions of a second-order algebraic equation, and the corresponding eigenfunctions are expressed in terms of generalized Laguerre polynomials. We presented a detailed study of some interesting particular cases of stationary states paying special attention to the differences between vector and scalar couplings. Furthermore, we show that when the vector and scalar couplings have the same magnitude the scattering amplitude for bosons (antibosons) is strongly suppressed when $g_{v}=-g_{s}\left(g_{v}=+g_{s}\right)$ and $|E| \simeq m c^{2}$. The analysis of eigenenergies for $g_{v} \simeq-g_{s}$ and $g_{v} \simeq+g_{s}$ showed that the accidental degeneracy seen in the cases $g_{v}=-g_{s}$ and $g_{v}=+g_{s}$ is broken perturbatively and that this breaking is 
related to the nonconservation of the Runge-Lenz vector.

It seems that the partial wave analysis for the elastic scattering of fermions by a Coulomb field with a general mixing of vector and scalar couplings deserves special attention with focus on the possible strong suppression of the scattering amplitude for antiparticles (particles) in the case of spin (pseudospin) symmetry in the low-momentum limit. The presence of an attractive scalar field in addition to the vector field allows to approach mesonic atoms with a very large $Z$ nucleus and this fact rises hope for approaching the spectroscopy of mesonic atoms with more realistic fields with the scalar-vector coupling scheme.

\section{ACKNOWLEDGMENTS}

This work was supported in part by means of funds provided by CAPES and CNPq. The authors would like to thank the referee for constructive criticism and valuable suggestions.

[1] W. Gordon, Z. Phys. 48, 180 (1928).

[2] N. F. Mott, Roy. Soc. Proc. (London) A 118, 542 (1928).

[3] N. F. Mott, Roy. Soc. Proc. (London) A 124, 425 (1929).

[4] I. J. Kang and L. M. Brown, Phys. Rev. 128, 2828 (1962).

[5] J. H. Hetherington, J. Math. Phys. 4, 357 (1963).

[6] G. H. Rawitscher, Phys. Lett. 9, 337 (1964).

[7] R. A. Eisenstein and F. Tabakin, Comp. Phys. Comm. 12, 237 (1976); M. D. Cooper and M. B. Johnson, Nucl. Phys. A 260, 352 (1976); M. D. Cooper, M. B. Johnson, and G. B. West, Nucl. Phys. A 292, 240 (1977); R. Jäckle, H. Pilkuhn, and H. G. Schlaile, Phys. Lett. B 76, 177 (1978).

[8] R. A. Eisenstein and F. Tabakin, Comp. Phys. Comm. 8, 130 (1974).

[9] M. D. Cooper, R. H. Jeppesen, and M. B. Johnson, Phys. Rev. C 20, 696 (1979).

[10] R. D. Hill, J. H. Hetherington, and D. G. Ravenhall, Phys. Rev. 122, 267 (1961).

[11] G. Jansen, M. Pusch, and G. Soff, Z. Phys. D 8, 315 (1988).

[12] W. Fleischer and G. Soff, Z. Naturforsch. A 39, 703 (1984).

[13] W. Greiner, B. Müller, and J. Rafelski, Quantum Electrodynamics of Strong Fields (Springer- 
Verlag, New York, 1985).

[14] C. Y. Chen, C. L. Liu, F. L. Lu, and D. S. Sun, Acta Phys. Sinica 52, 1579 (2003); Z. Q. Ma,

S. H. Dong, X. Y. Gu, J. Yu, and M. Lozada-Cassou, Int. J. Mod. Phys. E 13, 597 (2004).

[15] C. Y. Chen, D. S. Sun, and F. L. Lu, Phys. Lett. A 330, 424 (2004).

[16] G. B. Smith and L. J. Tassie, Ann. Phys. (NY) 65, 352 (1971).

[17] G. Soff, B. Müller, J. Rafelski, and W. Greiner, Z. Naturforsch. A 28, 1389 (1973).

[18] S. Hama, B. C. Clark, E. D. Cooper, H. S. Sherif, and R. L. Mercer, Phys. Rev. C 41, 2737 (1990).

[19] J. S. Bell and H. Ruegg, Nucl. Phys. B 98, 151 (1975).

[20] J. N. Ginocchio, Phys. Rep. 414, 165 (2005).

[21] P. R. Page, T. Goldman, and J. N. Ginocchio, Phys. Rev. Lett. 86, 204 (2001).

[22] S. G. Zhou, J. Meng, and P. Ring, Phys. Rev. Lett. 91, 262501 (2003); R. Lisboa, M. Malheiro, P. Alberto, M. Fiolhais, and A. S. de Castro, Phys. Rev. C 81, 064324 (2010).

[23] J. B. Bowlin, A. S. Goldhaber, and C. Wilkin, Z. Phys. A 331, 83 (1988).

[24] H. Leeb and S. A. Sofianos, Phys. Rev. C 69, 054608 (2004).

[25] J. N. Ginocchio, Phys. Rev. Lett. 82, 4599 (1999); H. Leeb and S. Wilmsen, Phys. Rev. C 62, 024602 (2000); J. Y. Guo, R. D. Wang, and X. Z. Fang, Phys. Rev. C 72, 054319 (2005); B. N. Lu, E. G. Zhao, and S. G. Zhou, Phys. Rev. Lett. 109, 072501 (2012); B. N. Lu, E. G. Zhao, and S. G. Zhou, Phys. Rev. C 88, 024323 (2013).

[26] P. Alberto, A. S. de Castro, and M. Malheiro, Phys. Rev. C 87, 031301 (2013).

[27] P. Alberto, A. S. de Castro, and M. Malheiro, Phys. Rev. C 75, 047303 (2007).

[28] G. Baym, Lectures on Quantum Mechanics (Westview, New York, 1969).

[29] Handbook of Mathematical Functions, edited by M. Abramowitz and I. A. Stegun (Dover Publications, New York, 1972).

[30] W. Pauli, Z. Phys. 36, 336 (1926); W. H. Heintz, Am. J. Phys. 44, 687 (1976); T. Yoshida, Am. J. Phys. 55, 1133 (1987); T. Yoshida, Nuovo Cimento B 103, 127 (1989).

[31] Q. G. Lin, Am. J. Phys. 68, 1056 (2000).

[32] C. E. Aguiar and M. F. Barroso, Am. J. Phys. 64, 1042 (1996). 